\title{
Animal Model of Dermatophytosis
}

\author{
Tsuyoshi Shimamura, ${ }^{1,2}$ Nobuo Kubota, ${ }^{1}$ and Kazutoshi Shibuya ${ }^{2,3}$ \\ ${ }^{1}$ ReD Laboratories, POLA Pharma Inc, 560 Kashio-cho, Totsuka-ku, Yokohama 244-0812, Japan \\ ${ }^{2}$ Department of Surgical Pathology, Toho University School of Medicine, 6-11-1 Omori-Nishi, Ota-Ku, Tokyo 143-8541, Japan \\ ${ }^{3}$ Department of Dermatology, Peking University First Hospital, Beijing 100034, China
}

Correspondence should be addressed to Kazutoshi Shibuya, kaz@med.toho-u.ac.jp

Received 9 December 2011; Revised 8 February 2012; Accepted 13 February 2012

Academic Editor: Monica Fedele

Copyright ( $\odot 2012$ Tsuyoshi Shimamura et al. This is an open access article distributed under the Creative Commons Attribution License, which permits unrestricted use, distribution, and reproduction in any medium, provided the original work is properly cited.

\begin{abstract}
Dermatophytosis is superficial fungal infection caused by dermatophytes that invade the keratinized tissue of humans and animals. Lesions from dermatophytosis exhibit an inflammatory reaction induced to eliminate the invading fungi by using the host's normal immune function. Many scientists have attempted to establish an experimental animal model to elucidate the pathogenesis of human dermatophytosis and evaluate drug efficacy. However, current animal models have several issues. In the present paper, we surveyed reports about the methodology of the dermatophytosis animal model for tinea corporis, tinea pedis, and tinea unguium and discussed future prospects.
\end{abstract}

\section{Introduction}

Superficial mycoses affect $20 \%$ to $25 \%$ of the world's population, and the incidence is increasing [1]. However, the mechanisms of the characteristic pathology, the mechanisms of host protection against infection, and the reason for intractable tinea pedis have not been clarified. Thus, experiments to elucidate these questions regarding dermatophytosis are needed using an animal model that closely resembles human pathology.

However, the suggestion has been made that the model of experimental infection on the backs of animals was insufficient to clarify the pathology of tinea corporis and tinea pedis because the site of infection was as severe as in kerion celsi, and the treatment duration was limited due to spontaneous healing within 4 weeks after infection [2]. Concerning onychomycosis, tinea unguium research in humans has not progressed due to the inability to biopsy nails. And the pathogenesis and the invasion have not been fully understood. Thus, there are few reports of a model of onychomycosis because of the difficulty in setting up a model.

In this paper, we surveyed the reported animal models of tinea corporis, tinea pedis, and tinea unguium and will discuss the future direction of research to establish a dermatophytosis animal model with high reproducibility for human dermatophytosis.

\section{Animal Model of Tinea Corporis}

Tinea corporis in humans is mainly caused by Trichophyton rubrum, Trichophyton mentagrophytes, and Microsporum canis and take several clinical forms but commonly present as classic ringworm [3]. Ringworm of the body is usually observed on the trunk, shoulders, or limbs, and occasionally the face (excluding the beard area). The infection may range from mild to severe, commonly appearing as annular, scaly patches with sharply marginated, raised erythematous vesicular borders [4].

The efforts to establish an animal model of tinea corporis began with the first report by Bloch in 1908 [5]. Sakai et al. [6] were the first to report of animal studies evaluating the efficacy of tinea corporis, and the research became the basis for the tinea corporis model. Subsequently, many research studies have been reported an animal model of tinea corporis since 1962. To reproduce dermatophytosis in humans, the examination of methods using animal models mainly focused on animal species, fungal selection, and the condition of the infection (occlusion or open condition with or without abrasion) of the skin.

2.1. Animal Species. The tinea corporis model has been reported in a variety of different animals, such as cockscomb 
[7], germ-free guinea pigs [8], grafting guinea pig skin onto congenitally athymic mice [9], mice $[10]$, nude rats $[11,12]$, rabbits [13], chickens [13], humans [14-16], and guinea pigs $[6,7,9,10,12,13,17-60]$.

According to reports of T. mentagrophytes on cockscomb and guinea pigs [7], the skin of guinea pigs showed a greater resemblance to human skin; however, cockscomb was more suitable than the skin of guinea pigs for fungal growth. Nonetheless, topical treatment of cockscomb with drugs is difficult, especially a fluid excipient.

In germfree guinea pigs infected with T. mentagrophytes, forming serious ulcers took twice the time as healing compared with conventionally reared guinea pigs. Cutaneous reinfection of germfree guinea pigs with $T$. mentagrophytes was protracted. However, the lesions healed in about the same time as the primary infection in conventionally reared guinea pigs. These reactions were considered cell-mediated hypersensitivity similar to contact dermatitis [8]. Thus, no advantage was gained in using germfree guinea pigs as the animal model for tinea corporis because tinea corporis in humans shows no spontaneous healing.

The in vivo model using nude mice xenografted with guinea pig skin showed well-grown dermatophytosis on the xenograft but not on nude mouse skin [9]. On the other hand, T. mentagrophytes could be cultured from the skin of nude rats $(\mathrm{rnu} / \mathrm{rnu})$ for 90 days and euthymic rats $(\mathrm{rnu} /+)$ for 35 days [11]. In these studies, the same strain of T. mentagrophytes ATCC18748 was used. Thus, this difference in the infection period between nude mice and nude rats was not due to the organism. We suggest that this difference involved the different structures of the skin. Fujita [61] reported that hairless guinea pigs whose skin resembled human hairbearing skin could be induced to show clinical signs similar to human hair-bearing skin lesions and suggested that the difference in the skin structure, especially the density of hair and the thickness of the horny cell layer, affected the establishment of the infection.

Infectivity of two strains of human origin, Trichophyton quinckeanum NCPF309 and T. mentagrophytes MRL 81/889, were evaluated in seven inbred strain mice: $B A L B / c, A K R$, $\mathrm{C} 3 \mathrm{H}, \mathrm{DBA} / 2,(\mathrm{CBA} \times \mathrm{DBA} / 2) \mathrm{F}_{1}, \mathrm{CBA}$, and C57BL/6 [10]. As a result, variations were noted in susceptibility in different inbred mice strains, and $\mathrm{BALB} / \mathrm{c}$ mice showed greater susceptibility to the infection from both fungal strains.

Ringworm lesions developed in rabbits infected with T. mentagrophytes B32663 (dog origin) after intravenous administration in the same manner as lesions that developed after percutaneous administration [13].

In experimental infections of humans [15], T. mentagrophytes ATCC18748 was applied to the ankles followed by 4day-continuous occlusion. The time course of the disease was described in fine details as follows. (1) During the first 2 days following removal of the occlusive patches, no signs of infection were present. (2) Erythema, edema, and small vesicles appeared and the degree of inflammation steadily increased from the 3rd to the 10th days. (3) The lesions enlarged to fill nearly the entire area originally overlain by the occlusive patch from the 11 th to the 22nd days. (4) The erythema and edema were replaced by scaling, and discrete follicular infections became apparent from the 23rd to the 45th days. (5) Finally, the infections healed spontaneously from the 46th to the 90th days. Furthermore, the volar surface of the forearm was infected using the same method. Pathological changes were the same as the lesions on the ankles. However, the whole infection period was shortened, and the lesions finally healed spontaneously within 60 days. The sustainability of lesions may involve the skin structure as well as immunity because involvement of immunity on sustainability of the disease in nude rats $(r n u / r n u)$ was clear from the difference in the duration of complete healing using euthymic rats $(\mathrm{rnu} /+)$ [11].

Guinea pigs have been widely used as a model due to reproducibility, high susceptibility, and its easy handling. However, different studies have been attempted because of the suggestion that there were defects wherein the clinical signs and the course of the pathology have not been similar to that of humans [62].

2.2. Selection of Fungi. In the preparation of a guinea pig model, the following fungi were examined: T. mentagrophytes, Trichophyton mentagrophytes var. quinckeanum (mice, T. mentagrophytes is synonymous), Trichophyton mentagrophytes var. granulosum (rodents, now designated Trichophyton interdigitale), Trichophyton mentagrophytes var. mentagrophytes (now designated T. interdigitale), Trichophyton mentagrophytes var. erinacei (hedgehog), Trichophyton verrucosum (cattle, horse), T. rubrum (anthropophilic), Trichophyton tonsurans (anthropophilic), Trichophyton asteroids, Trichophyton violaceum (anthropophilic), Trichophyton concentricum (anthropophilic), M. canis, Microsporum gypseum, Microsporum pesicolor, Candida albicans, and Epidermophyton floccosum. In general, zoophilic dermatophytes are more pathogenic to laboratory animals than anthropophilic strains $[63,64]$. Thus, T. mentagrophytes and M. canis were specially used for the study of susceptibility to infection $[2,61,63]$.

2.3. Pre- and Posttreatment. In addition to the selection of fungi, pre- or posttreatment included occlusion and abrasion $[23,34,37,38,42,47,51,56]$. Each occlusion method after inoculation of fungi was reported as follows: covered with ointment [56], covered by a sheet of polyethylene film with an impermeable plastic tape for $24 \mathrm{hr}$ [34], moistened gauze pad with Teflon between $24 \mathrm{hr}$ and $72 \mathrm{hr}$ [38, 39, 42], covered with a glass fiber filter [37], or covered with a chamber [51]. The occlusion method was suitable for the tinea corporis model because it was closer to natural infection and achieved high reproducibility [61]. On the other hand, Kerbs et al. reported that the occlusive method had not been implemented in the guinea pig model with $T$. mentagrophytes ATCC18748 because occlusion treatment weakened the infection [42]. Furthermore, Saunte et al. [51] suggested that inoculation under occlusion showed no advantage in the establishment of experimental infections. In consideration of these reports, we should consider the need for occlusion to infect fungi according to the procedure and fungal strains.

While there are some reports using nonabraded skin without occlusion $[44,53,55]$, abraded skin was used in 
many experiments to secure a high infection rate. The methods of abrading back skin include the use of sandpaper [22, $23,25,31,33,45,47,48,54,58]$, a roughened pestle [40, 41], a steel brush [50], pumice stone [17], shaving [51, 57, 59], and tape stripping $[21,26-30,32,35,36,46,51,52]$. The abrasion techniques using sandpaper, a roughened pestle, and a pumice stone are simple and easy; however, such techniques may lead to skin wounds such as an ulcer, which subsequently can be confused with lesions produced during dermatophyte infections [51]. Meanwhile, shaving and tape stripping avoids the problem of creating ulcers by an artificial procedure and can make the skin equally and superficially traumatized prior to inoculation [51]. In addition, Saunte et al. stated that pretreatment, such as shaving with a razor or tape stripping, did not influence the nature of the infection by $M$. canis [51].

2.4. Inoculation Size. An animal model using the occlusion method without abrasion may be made with a small amount of fungi (100 spores $\left./ 3.8 \mathrm{~cm}^{2}\right)[39,42]$. One report stated that the optimal conditions for dermatophytosis in guinea pigs were found to be an inoculum of $10^{7}$ fungal cells applied to abraded skin without occlusion and that of $10^{8}$ fungal cells induced severe lesions [47]. In almost all cases, the inoculum of $10^{7}$ fungal cells was applied to the area of infection made by dermatophytosis in vivo by all kinds of abrasion methods without occlusion.

2.5. Preparation Method of Tinea Corporis in Guinea Pigs Model. The typical methods of infection using T. mentagrophytes are described below, which summarized the results from a survey of the literature.

The nonocclusion method involved removing the back hair of guinea pigs from the entire surface of the skin with electric hair clippers and then preparing the inoculation site. Next, the skin was abraded by applying and removing adhesive tape three or four times, shaving the infection area using a razor, or abrasion with sandpaper, a roughened pestle, a steel brush, or a pumice stone. After pre-treatment to create an abrasion, the skin was inoculated by applying a volume of $50-100 \mu \mathrm{L}$ of $T$. mentagrophytes $10^{6}$ cells $/ \mathrm{mL}$. However, the application volume must be changed according to the area.

The occlusion method involved applying 100 cells/site to a section of the skin to induce an infection $[39,42]$ and did not include the abrasion procedure [2]. The duration of occlusion was set from $24 \mathrm{hr}$ to $72 \mathrm{hr}[34,37-39,51]$.

2.6. Pathology of Tinea Corporis in Guinea Pig Model. The pathophysiologic changes over time as a model of infection in the guinea pig showed that the normal evolution of dermatophytosis may be clearly divided into four phases [63]: incubation phase, spreading phase, inflammation phase, and healing or cleaning phase extending from days 25 to 60 . The length of each phase depends on the mode of infection, fungal species, and strain [63].

Fujita [61] described the pathophysiology of changes over time focusing on the biological responses of guinea pigs infected with T. mentagrophytes as the tinea corporis model.
Briefly, erythema and red papules appeared between 4 and 6 days after inoculation, and then the reaction to trichophytin was positive between 7 and 8 days. This positive reaction to trichophytin indicated the establishment of cellular immunity to T. mentagrophytes. Therefore, the erythema and papules gradually increased in size and then fused together. The lesions that climaxed at 14-15 days showed infiltrative plaque-like erythema with thick scale and crusts. Subsequently, these eruptions gradually disappeared and almost healed spontaneously within 4 weeks after inoculation. Another report by Koga [64] described the pathophysiologic changes over time as almost the same as stated above. Furthermore, Koga reported histopathological changes in detail: no findings except slight acanthosis thought to be formed by abrasion on the 3rd day after inoculation. Fungal elements were only confirmed in the horny cell layer of the epidermis and epithelium of the follicular infundibulum. Acanthosis and hyperkeratosis were noted over the entire surface of the skin, and vasodilatation and inflammatory cell infiltration in the upper layer of the dermis were observed on the 7th day. In addition, there was a large amount of hyphae in the horny cell layer of the epidermis. On the 14th day, spongiosis was observed along with severe inflammatory cell infiltration in the dermis, however, hyphae were present only in the follicles and not in the horny cell layer of the epidermis. This disappearance of hyphae in the horny cell layer of the epidermis was understood as a result of exclusion by increased skin turnover. In addition, hyphae in the follicles were excluded before and after the 20th day as part of spontaneous healing.

\subsection{Evaluation of Treatment and Prophylaxis by Tinea Cor-} poris Model. The tinea corporis animal model was used to estimate the efficacy of antifungal agents like bifonazole [21, $22,25,26,35,46,52]$, clotrimazole [22, 23, 25, 35, 37, 40, $46,52]$, miconazole [37, 44], tolnaftate [12, 22, 25, 37, 40], griseofulvin $[28,29,37,40,65]$, ketoconazole $[12,18,20$, $29,37,40]$, omoconazole $[32,36]$, terbinafine $[26,29,40$, $41,47,48,50,58,65]$, econazole [40], butenafine [22, 23, 33], naftifine [22], itraconazole [20, 27, 41, 50, 51, 59, 65], amorolfine [21, 65], sertaconazole [44], saperconazole [24], latoconazole [25], fluconazole [27, 28, 31, 41, 65], SCH 39304 [31, 54], lanoconazole [30, 33, 48], mupirocin [18], laudamonium [17], KP-103 [33], neticonazole [33], R126638 [59], voriconazole [57], ciclopirox [58], pramiconazole [50], luliconazole [48], and Astragalus verus Olivier [45].

In general, the timing of the start with oral or topical administration of antifungal agents is when the primary lesion begins to occur in each model or experiment. In most cases, the time is from 3-5 days after infection. In a recent study, Ghannoum et al. [47] started oral treatment of terbinafine on the day of infection considering pharmacokinetics. That is, the start of oral therapy with terbinafine was set to allow the drug to be absorbed by the gastrointestinal tract and to reach the tissue by the time the infection was established.

The prophylaxis effect of antifungal agents was evaluated in the tinea corporis model. There was a wide range in the start of administration between 5 days before infection and 
30 minutes after infection [12, 17, 22, 41, 59]. A clear rationale for when to start administration has not been described in detail in these reports. However, the start of administration and the duration of treatment should be set to consider the local skin pharmacokinetics of the drug. Although relapse after treatment was reported [33], examinations have not often been conducted because of spontaneous healing in the guinea pigs.

\section{Animal Model of Tinea Pedis}

The feet, especially the soles and toe webs, are the most frequent site of tinea pedis in humans. The most common clinical manifestation is intertriginous dermatitis, which presents with maceration, peeling, and fissuring in the spaces between the fourth and fifth toes. Another common presentation is the chronic, squamous, hyperkeratotic type in which fine silvery scales cover the pinkish skin of the soles, heels, and sides of the foot (moccasin foot). An acute inflammatory condition characterized by the formation of vesicles, pustules, and sometimes bullae is most frequently caused by $T$. mentagrophytes. The more chronic agents of tinea pedis is caused by T. rubrum, T. mentagrophytes var. interdigitale, and E. floccosum [4].

Fujita and Matsuyama reported an animal model of tinea pedis using a paper disk [66]. Meanwhile, Uchida and Yamaguchi reported another animal model using a sheer adhesive bandage [67]. These two animal models of tinea pedis in guinea pigs are superior because they showed clinical and histopathological nonspontaneous healing similar to human hyperkeratotic tinea pedis.

3.1. Selection of Fungi. In the first report on the establishment of an animal model, Fujita and Matsuyama examined the methodology in the fungal selection of T. mentagrophytes strains using the paper disk method, anthropophilic or zoophilic, and arthroconidia or microconidia [66]. As a result, no clear difference was noted between arthroconidia and microconidia by MFID $_{50}(50 \%$ minimal foot infectious dose). In the histopathological examination, both the anthropophilic and zoophilic strains infected the horny cell layer, and the fungal invasions apparently continued for 6 months. Furthermore, the zoophilic strain infected a deeper layer than the anthropophilic strain. Thus, the zoophilic strain is thought to be more suitable for the animal model than the anthropophilic strain.

3.2. Inoculation Size. No reports discussed a direct comparison using inoculation size in each animal model. Thus, we surveyed previously published reports using $T$. mentagrophytes TIMM2789 (SM-110), which was selected most often for the tinea pedis model.

The inoculation sizes in the paper disk method ranged from $5 \times 10^{4}$ to $5 \times 10^{6}$ fungal cells/planta $[66,68-72]$. In the bandage method, inoculation size was only $1 \times 10^{7}$ fungal cells/planta in all reports $[67,72-77]$. However, even if either method was selected, inoculum concentrations applied to the animal should be considered in the concept of the experiment described in the next section.

3.3. Duration of Infection and Treatment. The determination of the infection period can be divided into two major concepts. One is the concept of an infinitely close association with human pathology in order to accurately predict the clinical effects in humans. Another concept is that it should be evaluated easily and quickly as a screening method for candidate drugs. In the former, because it has been reported that it took more than 26 weeks to spread over the sole [66], the drug treatment was started 2 or 3 weeks after inoculation [73-76]. In this case, the duration of drug treatment had been set from 2 weeks to 4 weeks followed by the clinical application period. In the latter case, the drug treatment was started from 3 days after inoculation, and the duration of treatment was for 3 or 7 days $[71,72]$. In addition, the duration of being fixed by paper disk or bandage on the planta pedis was 3 or 7 days in both cases.

3.4. Preparation Method of Tinea Pedis. The paper disk method involved two types of paper disks, with or without aluminum foil. A paper disk with aluminum foil was covered with a piece of aluminum foil on one side $(1.5 \mathrm{~mm}$ thick by $8 \mathrm{~mm}$ in diameter), while the other side held the inoculum suspension. Any type of disc was wetted with $50 \mu \mathrm{L}$ of the inoculum suspension and then fixed on the planta pedis of the guinea pig foot with elastic adhesive tape. The disc was removed on the 3 rd or the 7 th day after infection.

The bandage method was as follows. Sheer Adhesive Bandages (Band-Aid; Johnson \& Johnson Co., Ltd., Tokyo, Japan) were wetted with $100 \mu \mathrm{L}$ of the inoculum suspension $\left(1 \times 10^{8}\right.$ conidia/mL) and then fixed on the sole of an animal's foot with elastic adhesive tape. The Sheer Adhesive Bandages were removed on the 3rd or the 7 th day after infection.

3.5. Pathology of Tinea Pedis Model. A detailed histopathological examination of the guinea pig model was reported by Fujita and Matsuyama [66]. With the anthropophilic strain T. mentagrophytes NTM-105, the infecting fungi were observed in the upper two-thirds of the horny cell layer. Vertical invasions to the deeper part of the horny cell layer next to the granular layer were not observed at any time. Thus, infection was superficial, and no inflammatory response was induced. The growing fungi spread horizontally in the horny cell layer, and all parts of the plantar side of the foot were infected. Clinical signs of erythema, hyperkeratosis, and desquamation were absent. Such a silent infection persisted throughout the observation period for 1 year.

In contrast to the anthropophilic strain T. mentagrophytes, the zoophilic strain T. mentagrophytes SM-110 invaded the whole horny cell layer, and infecting fungi were observed just above the granular layer. Vasodilatation in the dermal papillae was noted. Two weeks after inoculation, strong inflammatory responses were induced. Spongiosis and vesicle formation were characteristic changes in the 
epidermis. Infiltrations of mononuclear and polymorphonuclear cells were recognized under the vesicles and in the perivascular areas. Exocytosis of these cells into the spongiotic epidermis was also present. However, the strong inflammatory response subsided rapidly within a week, and mild responses persisted thereafter. Three weeks after inoculation, massive growth of fungi in the hair follicles and cellular infiltration became prominent in the skin of the dorsal foot area. Clinically, severe signs of indurated erythema and formation of thick scale and crust developed. All fungi were recovered by culture from the infected feet throughout the observation period for 6 months.

Although the anthropophilic strain T. mentagrophytes NTM-105 had spread only in a small area during 3 weeks, the infected area steadily spread to an approximately $19 \mathrm{~mm}$ radius at 13 weeks after inoculation. By contrast, the zoophilic strain T. mentagrophytes SM-110 spread rapidly at first, reaching sites approximately $8 \mathrm{~mm}$ from the point of inoculation within 4 weeks, but the subsequent rate of spread was much slower. Although the two strains spread differently, most areas of the plantar part were infected in 26 weeks.

3.6. Evaluation of Treatment with Tinea Pedis Model. Antifungal efficacy in the tinea pedis animal model was evaluated with different antifungals, such as butenafine $[73,78,79]$, latoconazole $[25,80]$, naftifine $[78]$, tolnaftate $[25,76,78$, $80]$, clotrimazole $[25,76,78,80]$, bifonazole $[25,74,78-$ 80], terbinafine [67, 71-73], griseofulvin [67], lanoconazole $[70-72,81]$, omoconazole [74], miconazole [75], NND502 (luliconazole) [72, 77], a combination drug of $0.2 \%$ pyrrolnitrin and $0.4 \%$ clotrimazole [75], variotin [76], phenyl-11-iodo-10-undecynoate [76], siccanin [76], KP-103 [81], and neticonazole [81]. Ohmi et al. compared antifungal efficacy with two animal models, tinea corporis and tinea pedis, using the same antifungal agents [80]. They suggested that the conventional tinea corporis model produced on the back of guinea pigs was sensitive to treatment with several major topical antifungal agents, such as clotrimazole, bifonazole, and tolnaftate, probably because of spontaneous healing during the experimental period. However, the tinea pedis model was much more resistant to treatment with these drugs. The mycological cure rate of bifonazole $1 \%$ cream in this report was $70 \%$. In the clinical report, that of bifonazole $1 \%$ cream for 4 weeks was $49.6 \%$ [82]. Thus, it is possible to compare the efficacy of each drug in tinea pedis model; however, it may not accurately reflect the clinical effectiveness yet due to the difference of mycological cure rate between human and animal.

3.7. Evaluation of Relapse by Tinea Pedis Model. The relapse after drug treatment was examined in the tinea pedis model, which would not have healed spontaneously, unlike the tinea corporis animal model, using the antifungal agents, such as butenafine [73, 79], bifonazole [79], terbinafine [73], KP-103 [81], neticonazole [81], lanoconazole [81], and NND-502 (luliconazole) [77]. The relapse of dermatophytosis, which is highly dependent on environmental factors, is difficult to determine accurately in clinical practice. Thus, verification of relapse using an animal model is very important research.

\section{Animal Model of Tinea Unguium}

Invasion of the nail plate by a dermatophyte is referred to as tinea unguium in humans. There are two main types of nail involvement: invasive subungual (distal and proximal) and superficial white mycotic infection (leukonychia trichophytica). T. rubrum and T. mentagrophytes, respectively, are the most common dermatophytes of this infection [4].

There are few reports of the animal model of tinea unguium. One study used guinea pigs $[73,83]$, and the other used rabbits [84]. This section describes the two animal models.

4.1. Animal Model of Tinea Unguium in Guinea Pigs. Uchida et al. reported that $T$. mentagrophytes could infect the nail when the tinea pedis model in guinea pigs would extend the duration of the experiment [73]. Subsequently, the tinea unguium model, which was modified by the above model, was reported and evaluated drug efficacy [83]. The method and the evaluation results of drug efficacy in tinea unguium using guinea pigs were as follows.

The arthrospores of T. mentagrophytes SM-110 were suspended, and the suspension was adjusted to give a concentration of $10^{8}$ spores $/ \mathrm{mL}$. Two paper disks were immersed by the fungal suspension and applied between the toes of the hind paw (between the second third toes and between the third and fourth toes) with a foam pad, then fixed with adhesive elastic tape (day 0 after infection). The disks were removed on day 21 after infection. The antifungal treatment, topical KP-103, amorolfine, and terbinafine and oral terbinafine, started on day 60 after infection when the invasion of T. mentagrophytes SM-110 into the nail was confirmed; treatment continued for 30 consecutive days. The therapeutic efficacy of tinea unguium was evaluated by the culture method to avoid the drug carryover effects by using dialyzed samples that have been digested by enzymes. In the result, topical amorolfine and topical or oral terbinafine were ineffective even in terms of reducing the fungal burden. In contrast, topical KP-103 significantly reduced the fungal burden in the infected nails compared with the burdens found in the vehicle- and oral-terbinafine-treated groups.

This model was able to evaluate drug efficacy by two administration routes, oral and topical. Furthermore, this model can prevent drug carryover in the recovery culture and assess the pure viability of the fungi. However, this evaluation method is applicable only to water-soluble drugs. Thus, further consideration in the case of lipid-soluble drugs is necessary.

4.2. Animal Model of Tinea Unguium in Rabbits. We established an animal model using rabbits with confirmed fungi in the deep layer of the nail under an immunosuppressive condition [84]. In this section, we describe the method and results of the drug efficacy of our model. 
In some preliminary studies, three points were concluded: first, microconidia were more suitable than arthroconidia with regard to infection rate; second, the postinfection period from the end of infection to nail sampling was needed to maintain high infection rates; and third, administration of an immunosuppressant was essential to make $T$. mentagrophytes invade the nail to establish a reproducible infection. Subsequently, we performed experiments aimed at setting the suitable postinfection period, and the protocol for this experiment was as follows.

The nails of rabbits were immunosuppressed with injections of methylprednisolone acetate intramuscularly prior to application of $0.2 \mathrm{~mL}$ of fungal suspension $\left(10^{8}\right.$ microconi$\mathrm{dia} / \mathrm{mL}$ ) of T. mentagrophytes TIMM2789 at a site between the lunula and the proximal nail fold. The nail plates of the first-to-third toes of the hind paw were wrapped together with a gauze patch and finger cot, and $0.5 \mathrm{~mL}$ of sterile water was injected into the finger cot to produce a culture environment around the nail that was seemed suitable for fungal growth. This condition was maintained for the duration of the infection for 2 weeks with no other intervention. The finger cot and the gauze patch were removed after 2 weeks of exposure, and this condition was maintained during for 0,2 , or 6 weeks without finger cot and gauze patch; this was termed the postinfection period. After each postinfection period was completed, the animals were sacrificed, and the nails were removed from the paw and treated histopathologically.

In the results, some of the infected nails became cloudy on gross appearance, which was similar to the findings with human onychomycosis. With a longer postinfection period, these findings were fully confirmed. On histopathological examination, hyphae of $T$. mentagrophytes penetrated the nail plate, and some invading fungi reached the nail bed. The infection rate in the sample at 0,2 , and 6 weeks after infection was $57 \%, 87 \%$, and $93 \%$, respectively. In addition, fungi proliferated and moved distally into the nail plate depending on the duration of infection. The presence of subungual abscess with associated necrosis of the epithelium of the nail bed or matrix was confirmed near the fungi in the nail plate. Above all, a high infection rate was obtained by 2 weeks inoculation with microconidia of T. mentagrophytes TIMM2789 and postinfection periods of more than 2 weeks were required.

Subsequently, the experiment for drug efficacy was confirmed for the topical antifungal agents, $8 \%$ ciclopirox nail lacquer and 5\% amorolfine nail lacquer, using this model. The therapeutic period was set as 4 weeks after a 2-week infection period. The animals in the untreated control group underwent the process of infection and removal of the material used for this process, but they were not exposed to the test agents. The next day after the last treatment, the animals were sacrificed and the nails were removed from the paw for histopathological and microbiological examinations. In the microbiological examination, the infected nail intended for evaluation using culture recovery was cut into 10 pieces in cross-sections, and each nail piece was cultured on Sabouraud dextrose agar for 2 weeks at $28^{\circ} \mathrm{C}$. A nail piece that had confirmed fungal growth was assessed as culture positive, and a nail with more than one culture-positive piece was considered fungus positive.

In the results of microbiological examination, a statistically significantly lower rate of culture positivity was found in the 5\% amorolfine nail lacquer group for comparing the infection rate to that in both the control and $8 \%$ ciclopirox nail lacquer groups. Additionally, when the rate of culture positivity in the drug-treated groups was subtracted from that in the nontreated group, the differences were $54.2 \%$ on $5 \%$ amorolfine nail lacquer and $8.3 \%$ on $8 \%$ ciclopirox nail lacquer. This figure was similar in the clinical reports [8587].

This is the first report of fungal behavior in the nail plate in an experimental animal model of onychomycosis. Our experimental animal model succeeded in encouraging T. mentagrophytes to invade the deeper layers of the nail plate. The findings in our model were similar to the clinical diagnoses of the proximal subungual type (PSO). Furthermore, the efficacy of this model was close to the clinical cure rate. Further research using this model may be able to clarify the pathogenesis of onychomycosis and contribute to the development of drugs that match the clinical efficacy.

\section{Approach to Accurately Evaluate the Antifungal Agent In Vivo}

Thus, animal models have been introduced to simulate human skin diseases, as well as changes in disease, and have presented different ideas for antifungal evaluation. It is also necessary to accurately evaluate the therapeutic effects that have been provided to create an environment in various pathological ways [88-90].

In evaluating the effectiveness of antifungal agents in samples from animals and humans, it is necessary to pay attention to the fact that the new medium can attenuate the effects of antifungal agents remaining in the skin sections as has been reported in in vitro experiments [88], as well as the culture results that have been reported in human skin sections [89]. In addition, methods have been reported to evaluate the effects of antifungal agents in accordance with the size of the expanding growth of fungal colonies [90]. Contributions have been made in the determination of in vivo effects of antifungal agents in combination with the previous medium has been suggested. Already a new method against onychomycosis was proposed by Nakashima et al. [91]. This approach is expected to be useful for evaluating the preliminary antifungal effect.

\section{Immunology}

An understanding of the defense mechanism of humans with regard to a fungus is very important in order to create a dermatophytosis animal model that shows high reproducibility of human disease. The major problem faced by animal models is the occurrence of spontaneous healing, which does not occur in humans.

Dermatophyte colonization on the human skin is characteristically limited to the dead keratinized tissue of the 
stratum corneum and results in a mild or intense inflammatory reaction. The fungi invade the horny layer which lacks a specific immune system to combat the infection are eliminated by skin turnover with subsequent desquamation. Nevertheless, both humoral and cell-mediated reactions and specific and nonspecific host defense mechanisms respond and eventually eliminate the fungus.

Detailed research on the relationship between fungi and immunity has been reviewed by Weitzman and Summerbell [4]. Briefly described below: (1) keratinases and glycopeptides are known as the two major classes of dermatophyte antigens. Keratinases, produced by the dermatophytes to enable skin invasion, elicit delayed-type hypersensitivity (DTH) responses when injected intradermally into the skin of animals. The protein portion of the glycopeptides preferentially stimulates cell-mediated immunity (CMI), whereas the polysaccharide portion preferentially stimulates humoral immunity. However, antibodies produced by humoral immunization do not help eliminate the infection since the highest level of antibodies is found in those patients with chronic infections. Rather, the development of CMI, which is correlated with DTH, is usually associated with a clinical cure and ridding the stratum corneum of the offending dermatophyte. (2) Infections by anthropophilic fungi like T. rubrum often elicit less of an inflammatory response and are less likely to elicit an intense DTH response than infections caused by geophilic or zoophilic dermatophytes which characteristically evoke an intense inflammatory reaction. Much of this inflammation is produced by the activated lymphocytes and macrophages which are involved in the DTH reaction to the trichophytin glycopeptides. (3) Enhanced proliferation of the skin in response to the inflammation may be the final mechanism that removes the fungus from the skin by epidermal desquamation. (4) Mannan, a glycoprotein component of the fungal cell wall, may suppress the inflammatory response especially in atopic or other persons susceptible to the mannan-induced suppression of CMI. Incubation of purified T. rubrum mannan (TRM) inhibited the lymphocyte proliferation response to mitogens and various antigenic stimuli. TRM also inhibits keratinocyte proliferation, thus slowing epidermal turnover and allowing for a more persistent chronic infection. (5) Chronic dermatophytosis may be caused by the anthropophilic form of $T$. mentagrophytes, T. mentagrophytes var. interdigitale (T. interdigitale). Primary chronic trichophytosis may be associated with defective phagocytosis of peripheral blood leukocytes and that this defect is probably caused by the fungus itself.

Keywords to note are keratinases, glycopeptides, CMI, DTH, Mannan, phagocytosis of peripheral blood leukocytes, and skin turnover. Thus, the factors of fungi such as keratinase and glycopeptides including Mannan induce human CMI which is specially correlated with DTH, phagocytosis of peripheral blood leukocytes, and inhibition of skin turnover. In other words, the loss or decrease of these host's defense mechanisms lead to unsuccessful elimination of the fungi and may cause chronic dermatophytosis. We suggest that future experiments will be required on the relationship between fungi and immunity to overcome the challenges of the current animal model.

\section{Animal Ethics}

Because reproduction of human pathophysiology is hardly difficult in an in vitro experiment, the experiments using animals are necessary for the elucidation of unknown pathogenesis and the development of significant drugs for patients. For now, animals will undergo invasive procedures and may bleed for several weeks after surgical intervention or another way in order to make a model of dermatophytosis which must be essentially regarded as a category of chronic inflammation. Thus, we will have to plan an experiment that should follow the spirit of the 3Rs: reduction, refinement, and replacement [92]. All experimental designs should be reviewed by the Institutional Animal Care and Use Committees (IACUC) to determine whether the protocol follows the experiment facility guidelines or national guidelines [93].

\section{Conclusion and Future Prospects}

Using the main dermatophytosis animal model for tinea corporis, tinea pedis, and tinea unguium to elucidate the pathophysiology of human dermatophytosis and to evaluate antifungal agent efficacy, various experiments have been conducted. Reports of the tinea corporis model have been most published as comparisons of other dermatophytosis models; however, a satisfactory model has not been reported with regard to reproducibility and usefulness in the evaluation of drug efficacy because of spontaneous healing. Thus, antifungal efficacy may be estimated as greater than the actual effect. For nonspontaneous healing, the tinea pedis model has been said that the best model for dermatophytosis. This model confirmed that drug efficacy was dependent on MIC values. On the other hand, intractable tinea pedis is observed in humans. The pathology of intractable tinea pedis cannot be reproduced in this model, yet. The eradication of onychomycosis is important because onychomycosis causes the recurrence of tinea pedis or the spread of infection. However, there is no animal model that can reproduce all types of tine unguium in humans.

These difficulties in the establishment of a dermatophytosis animal model are thought to be attributed to the differences between animals and humans: the skin structure, immune system, and causative fungi. The differences in the skin structure, such as the thickness of the epidermis and horny cell layer and the number of follicles, may determine whether fungi can attach to the skin surface because experimentally infection was performed on the xenografted guinea pig skin but not on the skin of xenograft nude mice. Complex immune functions against organisms through fungal attachment are activated in order to eliminate fungi by CMI involved in DTH and skin turnover. Thus, the spontaneous healing observed in the animal model is the host's normal response. Considering the situation that many challenges remain in animal models, however, the loss or decrease in normal immune functions may be necessary in order to create a reproducible animal model. The characteristics of the causative organisms are important in the animal model because the causes to be eliminated are antigens against metabolic products by fungi and a part of the fungus itself. 
With the rapid development of molecular biology in recent years has come the gradual expansion of genetic studies of Trichophyton spp. [94]. This body of research will enable the transformation of fungi. The creation of a transformed strain that causes lesions similar to humans, but also leads to understanding human disease, can lead to proper verification of the drug effect.

\section{Acknowledgments}

The authors would like thank Dr. Majima. This work was supported by the Health Science Research Grants for Research on Emerging and Re-emerging Infectious Diseases (H22-Shinkou-Ippan-8 and H23-Shinkou-Ippan-018), Measures for Intractable Diseases (H20-nannchi-ippann-35) from the Ministry of Health, Labor and Welfare of Japan; a grant from the Strategic Basis on Research Grounds for Nongovernmental Schools at Heisei 20th from the Ministry of Education, Culture, Sports, Science and Technology of Japan to K. S; a grant from the Strategic Research Foundation Grant-Aided Project for Private Schools at Heisei 23th from Ministry of Education, Culture, Sports, Science and Technology of Japan, 2011-2015. K. Shibuya received research grants from Pfizer Inc., Janssen Pharmaceutical K. K., and Dainippon Sumitomo Pharma Co. The authors declare that they have no competing interests.

\section{References}

[1] B. Havlickova, V. A. Czaika, and M. Friedrich, "Epidemiological trends in skin mycoses worldwide," Mycoses, vol. 52, no. 1, p. 95, 2009.

[2] S. Fujita, "Animal models of dermatomycoses in recent years," Japanese Journal of Medical Mycology, vol. 38, no. 1, pp. 33-37, 1997.

[3] S. J. Rupke, "Fungal skin disorders," Primary Care, vol. 27, no. 2, pp. 407-421, 2000.

[4] I. Weitzman and R. C. Summerbell, "The dermatophytes," Clinical Microbiology Reviews, vol. 8, no. 2, pp. 240-259, 1995.

[5] B. Bloch, "Zur lehre von den dermatomykosen," Archives of Dermatological Research, vol. 93, no. 1-2, pp. 157-220, 1908.

[6] S. Sakai, T. kada, G. Saito, N. Muraoka, and Y. Takahashi, "Studies on chemotherapy of trichophyton infection. 1. Antifungal properties of halogen phenol esters," Journal of the Scientific Research Institute, vol. 46, pp. 113-117, 1952.

[7] R. Sulzmann, W. Weuffen, and A. Kramer, "Testing of antimycoticly active substances on the model of experimental cockscomb dermatophytosis. 3. Comparative histological studies of mycosis foci of the cockscomb and of the back skin of the guinea pig," Mykosen, vol. 14, no. 1, pp. 15-18, 1971.

[8] F. Green III and E. Balish, "Trichophyton mentagrophytes dermatophytosis in germfree guinea pig," Journal of Investigative Dermatology, vol. 75, no. 6, pp. 476-480, 1980.

[9] F. Green III, K. W. Lee, and E. Balish, "Chronic T. Mentagrophytes dermatophytosis of guinea pig skin grafts on nude mice," Journal of Investigative Dermatology, vol. 79, no. 2, pp. 125-129, 1982.

[10] R. J. Hay, R. A. Calderon, and M. J. Collins, "Experimental dermatophytosis: the clinical and histopathologic features of a mouse model using Trichophyton quinckeanum (mouse favus)," The Journal of Investigative Dermatology, vol. 81, no. 3, pp. 270-274, 1983.

[11] F. Green, J. K. Weber, and E. Balish, "The thymus dependency of acquired resistance to Trichophyton mentagrophytes dermatophytosis in rats," Journal of Investigative Dermatology, vol. 81, no. 1, pp. 31-38, 1983.

[12] J. Weber and E. Balish, "Antifungal therapy of dermatophytosis in guinea pigs and congenitally athymic rats," Mycopathologia, vol. 90, no. 1, pp. 47-54, 1985.

[13] J. Van Cutsem and P. A. Janssen, "Experimental systemic dermatophytosis," Journal of Investigative Dermatology, vol. 83, no. 1, pp. 26-31, 1984.

[14] A. G. Knight, "A review of experimental human fungus infections," Journal of Investigative Dermatology, vol. 59, no. 4, pp. 354-358, 1972.

[15] J. H. Reinhardt, A. M. Allen, D. Gunnison, and W. A. Akers, "Experimental human Trichophyton mentagrophytes infections," Journal of Investigative Dermatology, vol. 63, no. 5, pp. 419-422, 1974.

[16] H. Nakajima, "The pathophysiology and defense mechanism against superficial and subcutaneous fungal infection," Japanese Journal of Medical Mycology, vol. 46, no. 1, pp. 5-9, 2005.

[17] A. Treiber, W. Pittermann, and H. C. Schuppe, "Efficacy testing of antimycotic prophylactics in an animal model," International Journal of Hygiene and Environmental Health, vol. 204, no. 4, pp. 239-243, 2001.

[18] R. O. Nicholas, V. Berry, P. A. Hunter, and J. A. Kelly, "The antifungal activity of mupirocin," The Journal of Antimicrobial Chemotherapy, vol. 43, no. 4, pp. 579-582, 1999.

[19] G. Maestrone, S. Sadek, and M. Mitrovic, "Lesions of dermatophytosis in quinea pigs treated with triamcinolone acetonide: an animal model," American Journal of Veterinary Research, vol. 34, no. 6, pp. 833-836, 1973.

[20] K. Uchida, H. Yamaguchi, and K. Shibuya, "The therapeutic effects of itraconazole, a new triazole antifungal agent, for experimental fungal infections," Japanese Journal of Antibiotics, vol. 44, no. 5, pp. 588-599, 1991.

[21] K. Uchida, A. Matsuzaka, and H. Yamaguchi, "Therapeutic effect of amorolfine on experimental dermatophytosis," The Japanese Journal of Antibiotics, vol. 44, no. 9, pp. 1020-1031, 1991.

[22] T. Arika, M. Yokoo, T. Hase, T. Maeda, K. Amemiya, and H. Yamaguchi, "Effects of butenafine hydrochloride, a new benzylamine derivative, on experimental dermatophytosis in guinea pigs," Antimicrobial Agents and Chemotherapy, vol. 34, no. 11, pp. 2250-2253, 1990.

[23] T. Arika, T. Hase, and M. Yokoo, "Anti-Trichophyton mentagrophytes activity and percutaneous permeation of butenafine in guinea pigs," Antimicrobial Agents and Chemotherapy, vol. 37, no. 2, pp. 363-365, 1993.

[24] K. P. Fu, D. M. Isaacson, J. Lococo, B. Foleno, and J. Hilliard, "In vitro and in vivo antidermatophytic activity of saperconazole a new fluorinated triazole," Drugs under Experimental and Clinical Research, vol. 18, no. 11-12, pp. 443-446, 1992.

[25] H. Oka, Y. Niwano, T. Ohmi, T. Tanake, M. Uchida, and H. Yamaguchi, "Therapeutic efficacy of latoconazole in formulations of clinical use on experimental dermatophytosis in guinea pigs," Arzneimittel-Forschung/Drug Research, vol. 42, no. 3, pp. 345-349, 1992.

[26] K. Uchida and H. Yamaguchi, "Therapeutic efficacy of terbinafine creams, a new allylamine antimycotic, in guinea pig dermatophytosis model," Japanese Journal of Medical Mycology, vol. 32, no. 4, pp. 333-342, 1991. 
[27] K. Nagino, H. Shimohira, M. Ogawa, K. Uchida, and H. Yamaguchi, "Comparison of the therapeutic efficacy of oral doses of fluconazole and itraconazole in a guinea pig model of dermatophytosis," Journal of Infection and Chemotherapy, vol. 6, no. 1, pp. 41-44, 2000.

[28] K. Nagino, H. Shimohira, M. Ogawa, K. Uchida, and H. Yamaguchi, "Comparison of the therapeutic efficacy of oral doses of fluconazole and griseofulvin in a guinea pig model of dermatophytosis," Journal of Antibiotics, vol. 53, no. 2, pp. 207-210, 2000.

[29] K. Uchida and H. Yamaguchi, "Therapeutic efficacy of oral administration of terbinafine in guinea pig trichophytosis model," Japanese Journal of Medical Mycology, vol. 34, no. 4, pp. 485-491, 1993.

[30] Y. Niwano, T. Tabuchi, K. Kanai, H. Hamaguchi, K. Uchida, and H. Yamaguchi, "Therapeutic efficacy of lanoconazole ointment in guinea pig model of tinea corporis, a comparative study with ointment and cream preparations," Japanese Journal of Antibiotics, vol. 48, no. 1, pp. 150-154, 1995.

[31] R. M. Parmegiani, D. Loebenberg, A. Cacciapuoti et al., "Sch 39304, a new antifungal agent: oral and topical treatment of vaginal and superficial infections," Journal of Medical and Veterinary Mycology, vol. 31, no. 3, pp. 239-248, 1993.

[32] T. Hashiguchi, A. Ryu, T. Itoyama, K. Uchida, and $H$. Yamaguchi, "Study of the effective dose of a topical antifungal agent, omoconazole nitrate, on the basis of percutaneous pharmacokinetics in guinea-pigs and mice," Journal of Pharmacy and Pharmacology, vol. 49, no. 8, pp. 757-761, 1997.

[33] Y. Tatsumi, M. Yokoo, T. Arika, and H. Yamaguchi, "KP-103, a novel triazole derivative, is effective in preventing relapse and successfully treating experimental interdigital tinea pedis and tinea corporis in guinea pigs," Microbiology and Immunology, vol. 46, no. 7, pp. 425-432, 2002.

[34] H. Tagami, S. Watanabe, and S. Ofuji, “Trichophytin contact sensitivity in guinea pigs with experimental dermatophytosis induced by a new inoculation method," Journal of Investigative Dermatology, vol. 61, no. 4, pp. 237-241, 1973.

[35] K. Uchida, S. Yamashita, and H. Yamaguchi, "Therapeutic effect of bifonazole, a topical imidazole antimycotic agent, on experimental Trichophyton mentagrophytes infection," Chemotherapy, vol. 32, no. 11, pp. 842-854, 1984.

[36] K. Uchida and H. Yamaguchi, "Oxiconazole nitrate, a new imidazole-antimycotic: evaluation of antifungal activity in vivo," Chemotherapy, vol. 32, no. 9, pp. 585-601, 1984.

[37] M. E. Valiant and B. M. Frost, "An experimental model for evaluation of antifungal agents in a Trichophyton mentagrophytes infection of guinea pigs," Chemotherapy, vol. 30, no. 1, pp. 54-60, 1984.

[38] J. H. Greenberg, R. D. King, S. Krebs, and R. Field, "A quantitative dermatophyte infection model in the guinea pig: a parallel to the quantitated human infection model," Journal of Investigative Dermatology, vol. 67, no. 6, pp. 704-708, 1976.

[39] S. Kerbs, J. Greenberg, and K. Jesrani, "Temporal correlation of lymphocyte blastogenesis, skin test responses and erythema during dermatophyte infections," Clinical and Experimental Immunology, vol. 27, no. 3, pp. 526-530, 1977.

[40] G. Petranyi, J. G. Meingassner, and H. Mieth, "Activity of terbinafine in experimental fungal infections of laboratory animals," Antimicrobial Agents and Chemotherapy, vol. 31, no. 10, pp. 1558-1561, 1987.

[41] H. Mieth, I. Leitner, and J. G. Meingassner, "The efficacy of orally applied terbinafine, itraconazole and fluconazole in models of experimental trichophytoses," Journal of Medical and Veterinary Mycology, vol. 32, no. 3, pp. 181-188, 1994.
[42] S. Kerbs and A. M. Allen, "Effect of occlusion on Trichophyton mentagrophytes infections in guinea pigs," Journal of Investigative Dermatology, vol. 71, no. 5, pp. 301-304, 1978.

[43] F. Green III, J. W. Anderson, and E. Balish, "Cutaneous basophil hypersensitivity and contact sensitivity after cutaneous Trichophyton mentagrophytes infection," Infection and Immunity, vol. 29, no. 2, pp. 758-767, 1980.

[44] C. Palacin, A. Sacristan, and J. A. Ortiz, "In vivo activity of sertaconazole in experimental dermatophytosis in guinea pigs," Arzneimittel-Forschung A, vol. 42, no. 5, pp. 714-718, 1992.

[45] A. Mikaeili, M. Modaresi, I. Karimi, H. Ghavimi, M. Fathi, and N. Jalilian, "Antifungal activities of astragalus verus olivier. against trichophyton verrucosum on in vitro and in vivo guinea pig model of dermatophytosis," Mycoses. In press.

[46] H. Yamaguchi and K. Uchida, "In vivo activity of bifonazole in guinea pigs: its characteristic features and comparison with clotrimazole," Dermatologica, vol. 169, supplement 1, pp. 3345, 1984.

[47] M. A. Ghannoum, M. A. Hossain, L. Long, S. Mohamed, G. Reyes, and P. K. Mukherjee, "Evaluation of antifungal efficacy in an optimized animal model of Trichophyton mentagrophytes-dermatophytosis," Journal of Chemotherapy, vol. 16, no. 2, pp. 139-144, 2004.

[48] M. A. Ghannoum, L. Long, H. G. Kim, A. J. Cirino, A. R. Miller, and P. Mallefet, "Efficacy of terbinafine compared to lanoconazole and luliconazole in the topical treatment of dermatophytosis in a guinea pig model," Medical Mycology, vol. 48, no. 3, pp. 491-497, 2010.

[49] X. J. Chen, Y. N. Shen, G. X. Lü, and W. D. Liu, "Establishing an experimental guinea pig model of dermatophytosis using Trichophyton rubrum," Acta Academiae Medicinae Sinicae, vol. 30, no. 5, pp. 599-602, 2008.

[50] K. de Wit, C. Paulussen, A. Matheeussen, K. van Rossem, P. Cos, and L. Maes, "In vitro profiling of pramiconazole and in vivo evaluation in Microsporum canis dermatitis and Candida albicans vaginitis laboratory models," Antimicrobial Agents and Chemotherapy, vol. 54, no. 11, pp. 4927-4929, 2010.

[51] D. M. Saunte, J. P. Hasselby, A. Brillowska-Dabrowska et al., "Experimental guinea pig model of dermatophytosis: a simple and useful tool for the evaluation of new diagnostics and antifungals," Medical Mycology, vol. 46, no. 4, pp. 303-313, 2008.

[52] K. Uchida and H. Yamaguchi, "Assessment of in vivo activity of bifonazole against dermatophytic infection in guinea pigs on the basis of the amount of a specific fungal cell wall component chitin in the infected skin," Dermatologica, vol. 169, supplement 1, pp. 47-50, 1984.

[53] Z. Hussin and J. M. Smith, "Vaccination procedures and the infectivity of dermatophyte lesions," Mycopathologia, vol. 81, no. 2, pp. 71-76, 1983.

[54] L. Wearley, B. Antonacci, A. Cacciapuoti et al., "Relationship among physicochemical properties, skin permeability, and topical activity of the racemic compound and pure enantiomers of new antifungal," Pharmaceutical Research, vol. 10, no. 1, pp. 136-140, 1993.

[55] N. Chittasobhon and J. M. Smith, "The production of experimental dermatophyte lesions in guinea pigs," Journal of Investigative Dermatology, vol. 73, no. 2, pp. 198-201, 1979.

[56] A. F. Bonk, L. Friedman, and V. J. Derbes, "Experimental dermatophytosis," The Journal of Investigative Dermatology, vol. 39, pp. 281-286, 1962. 
[57] D. M. Saunte, F. Simmel, N. Frimodt-Moller et al., "In vivo efficacy and pharmacokinetics of voriconazole in an animal model of dermatophytosis," Antimicrobial Agents and Chemotherapy, vol. 51, no. 9, pp. 3317-3321, 2007.

[58] M. A. Ghannoum, L. Long, and W. R. Pfister, "Determination of the efficacy of terbinafine hydrochloride nail solution in the topical treatment of dermatophytosis in a guinea pig model," Mycoses, vol. 52, no. 1, pp. 35-43, 2009.

[59] F. Odds, J. Ausma, F. Van Gerven et al., "In vitro and in vivo activities of the novel azole antifungal agent R126638," Antimicrobial Agents and Chemotherapy, vol. 48, no. 2, pp. 388-391, 2004.

[60] H. Yamaguchi and K. Uchida, "Once daily administration of terbinafine to guinea-pigs with experimental dermatophytosis," Clinical and Experimental Dermatology, vol. 14, no. 2, pp. 108-109, 1989.

[61] S. Fujita, "Conditions required for successful experimental dermatophytosis and its histopathogenesis," Japanese Journal of Medical Mycology, vol. 33, no. 2, pp. 115-125, 1992.

[62] K. Uchida and H. Yamaguchi, "Preclinical therapeutic evaluation of agents for treating dermatophytosis," Japanese Journal of Medical Mycology, vol. 37, no. 4, pp. 199-205, 1996.

[63] J. Van Cutsem, "Animal models for dermatomycotic infections," Current Topics in Medical Mycology, vol. 3, pp. 1-35, 1989.

[64] H. Koga, "Animal model for superficial mycosis," Japanese Journal of Medical Mycology, vol. 50, no. 2, pp. 84-89, 2009.

[65] A. Polak, "Combination of amorolfine with various antifungal drugs in dermatophytosis," Mycoses, vol. 36, no. 1-2, pp. 4349, 1993.

[66] S. Fujita and T. Matsuyama, "Experimental tinea pedis induced by non-abrasive inoculation of Trichophyton mentagrophytes arthrospores on the plantar part of a guinea pig foot," Journal of Medical and Veterinary Mycology, vol. 25, no. 4, pp. 203-213, 1987.

[67] K. Uchida and H. Yamaguchi, "Effectiveness of oral treatment with terbinafine in a guinea pig model of tinea pedis," Japanese Journal of Antibiotics, vol. 47, no. 10, pp. 1401-1406, 1994.

[68] S. Fujita, T. Matsuyama, and Y. Sato, "Experimental tinea pedis in guinea pig feet-scanning electron microscopic and histological study of the infection," Japanese Journal of Medical Mycology, vol. 29, no. 3, pp. 163-168, 1988.

[69] S. Fujita, Y. Kaneko, Y. Sato, and T. Matsuyama, "Invasiveness of infecting fungi and tissue response in experimental tinea pedis in guinea pigs," Japanese Journal of Medical Mycology, vol. 30, no. 4, pp. 233-240, 1989.

[70] Y. Niwano, T. Tabuchi, K. Kanai, H. Hamaguchi, K. Uchida, and H. Yamaguchi, "Therapeutic efficacy of lanoconazole ointment in guinea pig model of tinea pedis, in comparison with that of cream preparations," Japanese Journal of Antibiotics, vol. 47, no. 9, pp. 1192-1195, 1994.

[71] Y. Niwano, T. Tabuchi, K. Kanai, H. Hamaguchi, K. Uchida, and H. Yamaguchi, "Short-term topical therapy of experimental tinea pedis in guinea pigs with lanoconazole, a new imidazole antimycotic agent," Antimicrobial Agents and Chemotherapy, vol. 39, no. 10, pp. 2353-2355, 1995.

[72] Y. Niwano, N. Kuzuhara, H. Kodama, M. Yoshida, T. Miyazaki, and H. Yamaguchi, "In vitro and in vivo antidermatophyte activities of NND-502, a novel optically active imidazole antimycotic agent," Antimicrobial Agents and Chemotherapy, vol. 42, no. 4, pp. 967-970, 1998.

[73] K. Uchida, M. Kudoh, and H. Yamaguchi, "A study on effectiveness of treatment and prevention of relapse using topical administration of terbinafine in a guinea pig model for tinea pedis," The Japanese Journal of Antibiotics, vol. 47, no. 10, pp. 1407-1412, 1994.

[74] T. Itoyama, K. Uchida, H. Yamaguchi, and S. Fujita, "Therapeutic effects of omoconazole nitrate on experimental tinea pedis, an intractable dermatophytosis, in guinea-pigs," Journal of Antimicrobial Chemotherapy, vol. 40, no. 3, pp. 441-444, 1997.

[75] K. Uchida and H. Ymaguchi, "Therapeutic efficacy of a topical antifungal solution preparation formulated with pyrrolnitrin and clotrimazole in combination (Pyroace) in guinea pig model of tinea pedis," Japanese Journal of Antibiotics, vol. 52, no. 1, pp. 68-74, 1999.

[76] H. Yamaguchi, K. Uchida, T. Tanaka, and T. Yamaguchi, "Therapeutic efficacy of a topical tolnaftate preparation in guinea pig model of tinea pedis," The Japanese Journal of Antibiotics, vol. 54, no. 6, pp. 323-330, 2001.

[77] K. Uchida, T. Tanaka, and H. Yamaguchi, "Achievement of complete mycological cure by topical antifungal agent NND502 in guinea pig model of tinea pedis," Microbiology and Immunology, vol. 47, no. 2, pp. 143-146, 2003.

[78] T. Arika, M. Yokoo, T. Maeda, K. Ameniya, and H. Yamaguchi, "Effects of butenafine hydrochloride, a new benzylamine derivative, on experimental tinea pedis in guinea pigs," Antimicrobial Agents and Chemotherapy, vol. 34, no. 11, pp. 22542255, 1990.

[79] T. Arika, M. Yokoo, and H. Yamaguchi, "Topical treatment with butenafine significantly lowers relapse rate in an interdigital tinea pedis model in guinea pigs," Antimicrobial Agents and Chemotherapy, vol. 36, no. 11, pp. 2523-2525, 1992.

[80] T. Ohmi, S. Konaka, M. Uchida, and H. Yamaguchi, "Antifungal activity of the new agent latoconazole in two tinea models," Arzneimittel-Forschung/Drug Research, vol. 41, no. 8, pp. 847851, 1991.

[81] Y. Tatsumi, M. Yokoo, T. Arika, and H. Yamaguchi, "In vivo fungicidal effect of KP-103 in a guinea pig model of interdigital tinea pedis determined by using a new method for removing the antimycotic carryover effect," Microbiology and Immunology, vol. 46, no. 7, pp. 433-439, 2002.

[82] S. Watanabe, H. Takahashi, T. Nishikawa et al., "A comparative clinical study between 2 weeks of luliconazole $1 \%$ cream treatment and 4 weeks of bifonazole $1 \%$ cream treatment for tinea pedis," Mycoses, vol. 49, no. 3, pp. 236-241, 2006.

[83] Y. Tatsumi, M. Yokoo, H. Senda, and K. Kakehi, "Therapeutic efficacy of topically applied KP-103 against experimental tinea unguium in guinea pigs in comparison with amorolfine and terbinafine," Antimicrobial Agents and Chemotherapy, vol. 46, no. 12, pp. 3797-3801, 2002.

[84] T. Shimamura, N. Kubota, S. Nagasaka, T. Suzuki, H. Mukai, and K. Shibuya, "Establishment of a novel model of onychomycosis in rabbits for evaluation of antifungal agents," $A n-$ timicrobial Agents and Chemotherapy, vol. 55, no. 7, pp. 31503155, 2011.

[85] J. Lauharanta, "Comparative efficacy and safety of amorolfine nail lacquer $2 \%$ versus $5 \%$ once weekly," Clinical and Experimental Dermatology, Supplement, vol. 17, no. 1, pp. 41-43, 1992.

[86] D. Reinel and C. Clarke, "Comparative efficacy and safety of amorolfine nail lacquer $5 \%$ in onychomycosis, once-weekly versus twice-weekly," Clinical and Experimental Dermatology, Supplement, vol. 17, no. 1, pp. 44-49, 1992.

[87] A. K. Gupta and W. S. Joseph, "Ciclopirox 8\% nail lacquer in the treatment of onychomycosis of the toenails in the United States," Journal of the American Podiatric Medical Association, vol. 90, no. 10, pp. 495-501, 2000. 
[88] T. Nakashima, A. Nozawa, T. Ito, T. Majima, and H. Yamaguchi, "Development of a new medium useful for the recovery of dermatophytes from clinical specimens by minimizing the carryover effect of antifungal agents," Microbiology and Immunology, vol. 46, no. 2, pp. 83-88, 2002.

[89] M. Adachi and S. Watanabe, "Evaluation of combined deactivators-supplemented agar medium (CDSAM) for recovery of dermatophytes from patients with tinea pedis," Medical Mycology, vol. 45, no. 4, pp. 347-349, 2007.

[90] T. Majima, S. Masui, K. Uchida, and H. Yamaguchi, "A novel mycological analysis valuable for evaluating therapeutic efficacy of antimycotics against experimental dermatophytosis in guinea pigs," Mycoses, vol. 48, no. 2, pp. 108-113, 2005.

[91] T. Nakashima, A. Nozawa, T. Ito, and T. Majima, "Experimental tinea unguium model to assess topical antifungal agents using the infected human nail with dermatophyte in vitro," Journal of Infection and Chemotherapy, vol. 8, no. 4, pp. 331335, 2002.

[92] W. M. S. Russell and R. L. Burch, The Principles of Humane Experimental Technique, Methuen \& Co., London, UK, 1959.

[93] Institute of Laboratory Animal Resources-National Research Council, Guide for the Care and Use of Laboratory Animals, National Academy Press, Washington, DC, USA, 8th edition, 2011.

[94] T. Yamada, "Molecular approach to pathology of and immunity against dermatophytes," Japanese Journal of Medical Mycology, vol. 49, no. 4, pp. 293-297, 2008. 


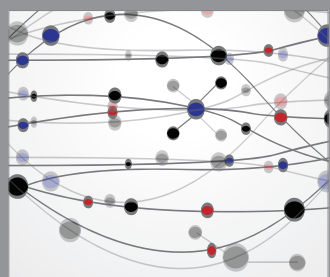

The Scientific World Journal
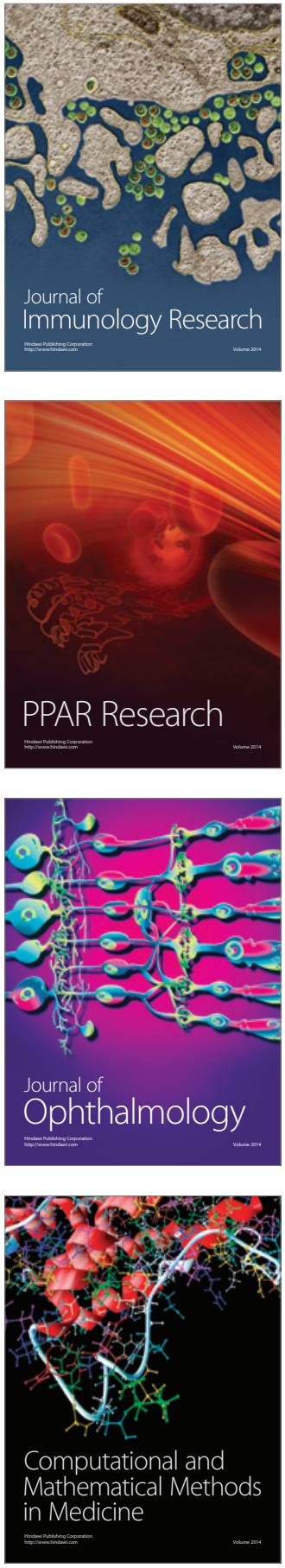

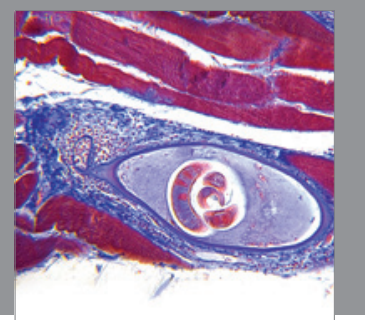

Gastroenterology

Research and Practice
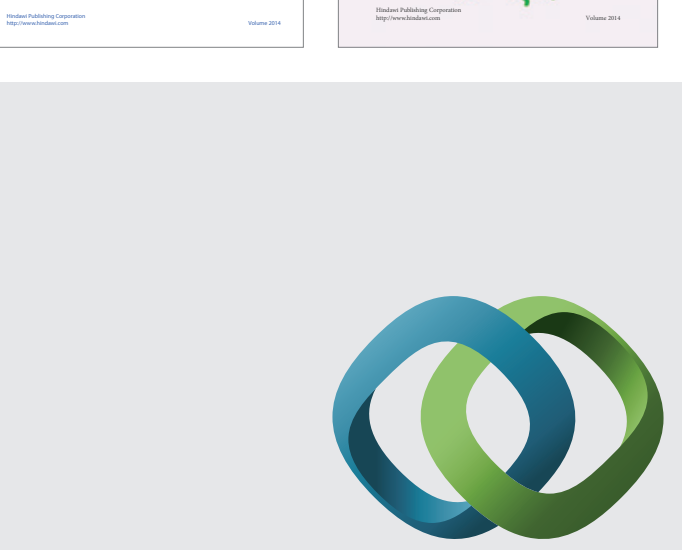

\section{Hindawi}

Submit your manuscripts at

http://www.hindawi.com
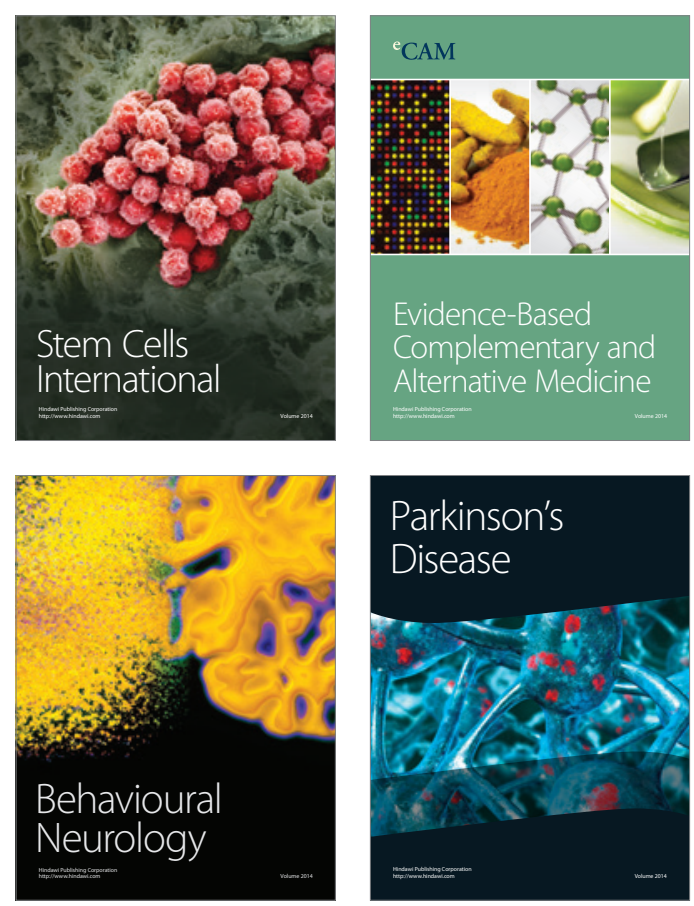

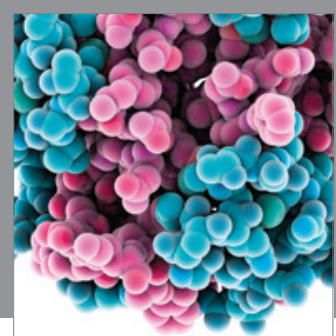

Journal of
Diabetes Research

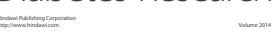

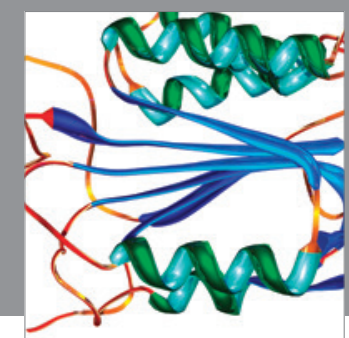

Disease Markers
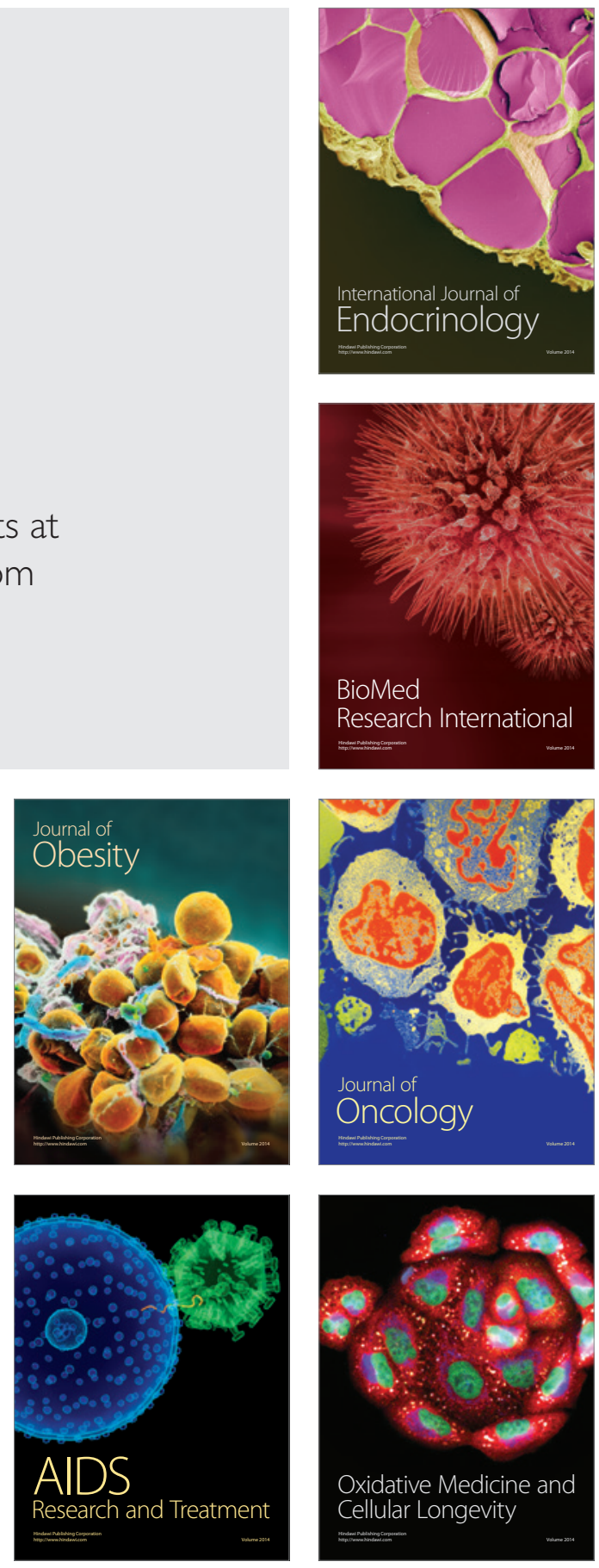